\section{Metastatic Cardiac Tumor Presenting as an Anteroseptal ST-Segment Elevation Myocardial Infarction in a Young Male}

\author{
John-Henry L. Dean ${ }^{1}$, Arjun G. Kalra ${ }^{1}$, Shayef Gabasha ${ }^{2}$, Rosco Gore ${ }^{2}$ \\ 1. Department of Internal Medicine, Brooke Army Medical Center, San Antonio, USA 2. Department of Cardiology, \\ Brooke Army Medical Center, San Antonio, USA
}

Corresponding author: John-Henry L. Dean, johnhenry_dean@yahoo.com
Review began 03/11/2021 Review ended 03/17/2021 Published 03/18/2021

\section{○ Copyright 2021}

Dean et al. This is an open access article distributed under the terms of the Creative Commons Attribution License CC-BY 4.0., which permits unrestricted use, distribution, and reproduction in any medium, provided the original author and source are credited.
Categories: Cardiology, Emergency Medicine, Internal Medicine

Keywords: st-segment elevation myocardial infarction (stemi), bone and soft-tissue sarcoma, metastatic tumor to the heart, pericardial tamponade

\section{Introduction}

Elevation of the ST-segment on electrocardiogram (ECG) typically generates a list of potential diagnoses comprising common etiologies (e.g., ST-segment elevation myocardial infarction [STEMI], early repolarization, pericarditis, and ST-segment elevation secondary to a primary depolarization abnormality such as left ventricular hypertrophy/left bundle branch block or pre-excitation), as well as less common etiologies (e.g., Brugada syndrome, hyperkalemia, and pulmonary embolism) [1]. Cardiac neoplasms represent a rare clinical entity [2,3], and as such, are infrequently considered in the differential diagnosis of ST-segment elevation. Several case reports have been published on ST-segment changes in the context of cardiac tumors, though the precise pathophysiological mechanism behind the ECG changes continues to be debated. Suggested hypotheses for these changes include embolization of tumor fragments to coronary arteries, external compression of coronary arteries, distension of cardiac muscle fibers, peri-tumor inflammatory reactions, and electrolyte transfer from necrotic tumor tissue to adjacent myocardium $[4,5]$. Regardless of the particular mechanism involved, most documented cases of ST-segment elevation in the setting of cardiac tumors often appear to present with chest pain/discomfort or clinical signs of developing heart failure [4,5]. Here, however, we describe the case of a patient who presented with ST-segment elevation in the absence of any cardiopulmonary symptoms.

\section{Case Presentation}

A 28-year-old, active-duty military male with relapsed, refractory, metastatic, malignant, giant-cell-rich, primary bone sarcoma presented to the Emergency Department (ED) by cardiology instruction for evaluation of a large pericardial effusion with possible early tamponade physiology that was identified on an outpatient transthoracic echocardiogram (TTE). The echocardiogram was performed to further evaluate findings from a positron emission tomography (PET) scan completed three days prior that demonstrated findings concerning for cardiac metastasis to the interventricular septum with extension into the right ventricular outflow tract, along with an interval increase in the size of a pericardial effusion when compared to a PET scan from five months prior (Figure 7 . At the time of initial evaluation in the ED, the patient was asymptomatic from a cardiopulmonary standpoint, specifically denying chest pain or dyspnea, though endorsing chronic superficial abdominal tenderness in multiple abdominal regions. Vital signs were normal and physical examination was significant only for mild epigastric and right upper quadrant tenderness. An ECG was obtained that demonstrated sinus rhythm, low voltage, and anteroseptal myocardial injury (Figure 2). 


\section{Cureus}

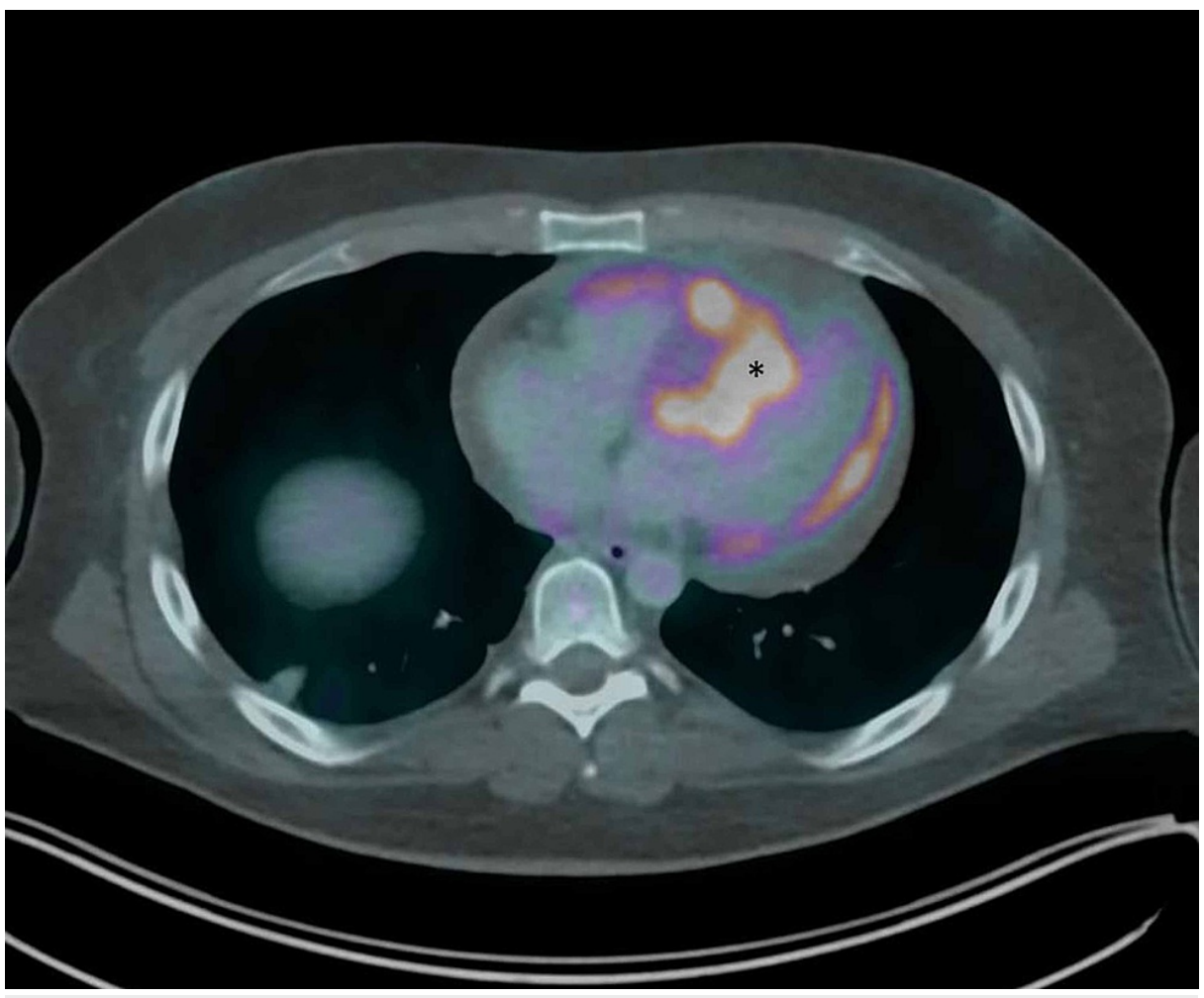

FIGURE 1: PET scan demonstrating area of increased radiotracer uptake and metabolic activity (asterisk) at the site of the cardiac tumor in the interventricular septum.

$\mathrm{PET}$, positron emission tomography

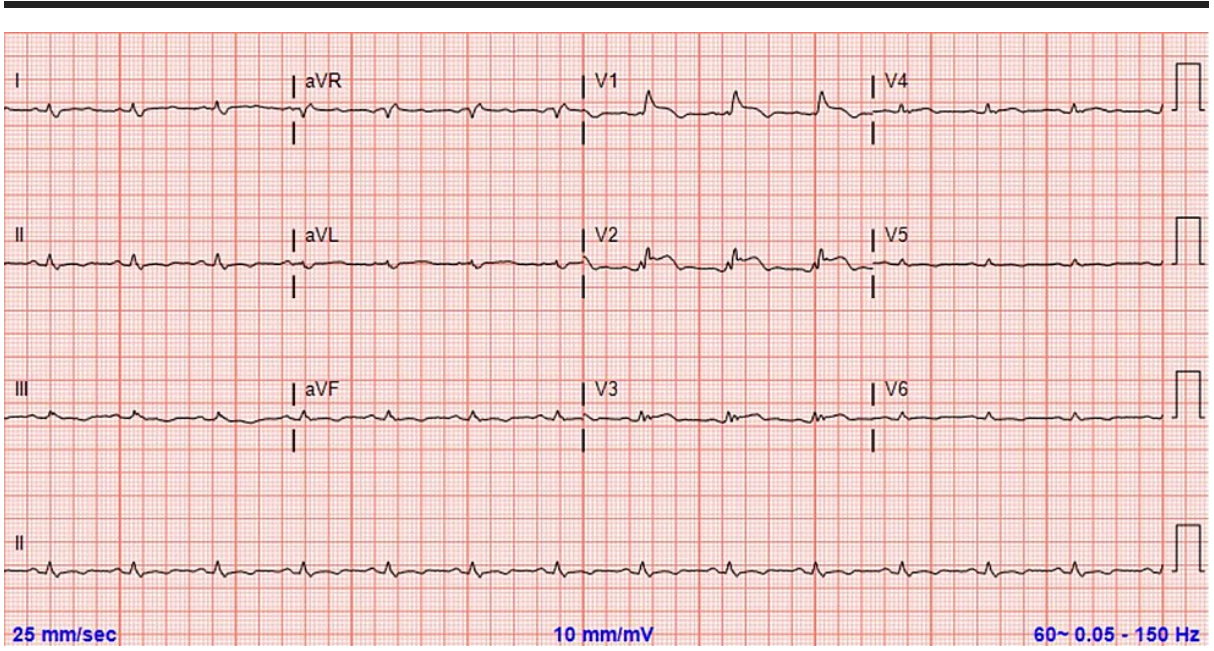

FIGURE 2: Initial ECG showing sinus rhythm, low voltage, and STsegment elevation in anteroseptal precordial leads.

ECG, electrocardiogram

In the setting of ST-segment elevation, additional concern was raised by the discovery of hypokinesis of the septal myocardium on bedside echocardiogram, though it should be noted that this area of myocardium was being impinged upon by a $47 \mathrm{~mm} \times 23 \mathrm{~mm}$ mass in the interventricular septum (Figure 3). 


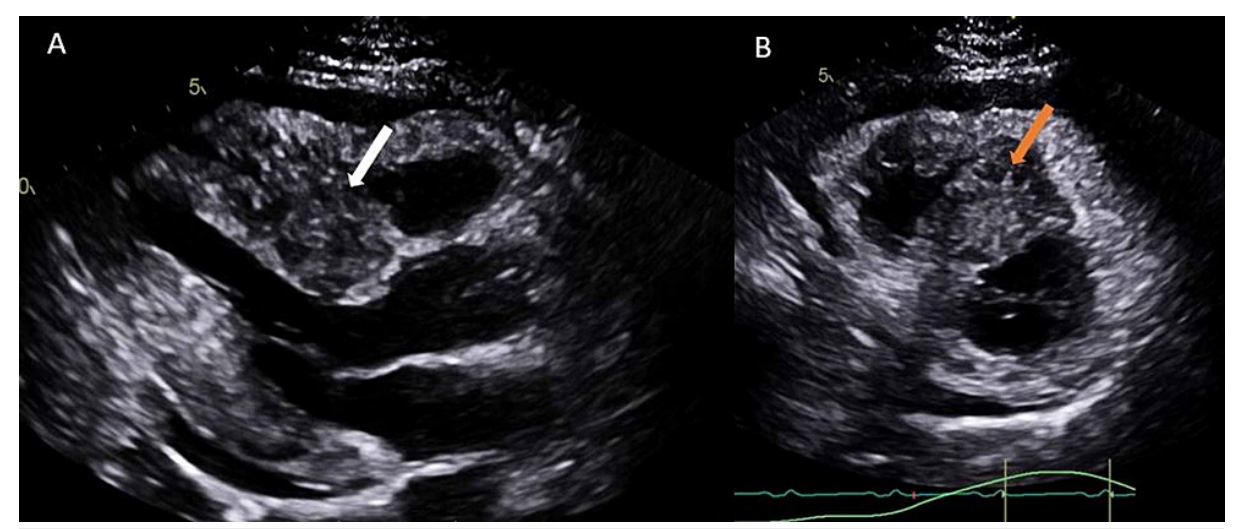

FIGURE 3: Two-dimensional TTE with parasternal long-axis view (A) showing hypoechoic mass (white arrow) in the interventricular septum and parasternal short-axis view (B) demonstrating spiculations (orange arrow) radiating out from the same central mass.

TTE, transthoracic echocardiogram

However, given the lack of cardiopulmonary symptoms, the patient was treated conservatively with serial ECGs and cardiac enzymes. Initial troponin was $0.14 \mathrm{ng} / \mathrm{mL}$ (99th percentile for assay being $0.03 \mathrm{ng} / \mathrm{mL}$ ) with stable values on repeat assays performed at six-hour intervals. The patient was then admitted to the Coronary Care Unit and monitored closely for signs of cardiac tamponade (i.e., pulsus paradoxus). He subsequently required pericardiocentesis for a large pericardial effusion concerning for tamponade physiology based on mitral inflow velocities. Based on cytology with negative infectious labs, the effusion was deemed secondary to his malignancy. Following a seven-day hospitalization and extensive multidisciplinary discussions with the patient and his family regarding the prognosis, the patient elected to forgo any further cardiac imaging and was discharged home with hospice, where he died shortly after discharge.

\section{Discussion}

We present a case of characteristic ST-segment elevation suggestive of anteroseptal STEMI in a patient with no risk factors for coronary artery disease, a recently identified cardiac mass, and without any correlating symptoms. Our patient's troponin T assay was found to be elevated above the 99th percentile of the upper reference limit on initial assessment without a subsequent rise and/or fall, and thus did not meet the criteria for myocardial infarction according to the Fourth Universal Definition of Myocardial Infarction [6]. Given that the patient was a young, active-duty male (a patient population with a low risk for atherosclerotic coronary disease making STEMI highly unlikely), and that the clinical picture was not suggestive of an acute coronary syndrome (no symptoms of myocardial ischemia), the decision was made to forgo cardiac catheterization. With the time-sensitive and life-threatening nature of STEMIs, we believe this case illustrates the importance of maintaining a suspicion for alternative diagnoses when STEMI does not fit the clinical picture as the decision to begin anticoagulation and perform a left heart catheterization can place the patient at higher risk with less potential benefit in these scenarios. After much thought and discussion, we surmised that the ECG findings were most likely attributable to a persistent current of injury related to the interventricular septal mass.

Various cases of patients with cardiac tumors presenting with ECG changes have been reported dating back to as early as 1934 [7]. We consider this case important to report because the patients in most of the previously documented cases presented with cardiopulmonary symptoms. For example, a case reported by Demir et al. in January 2019 described a 59-year-old man with a history of tongue cancer who presented to the emergency department for acute-onset chest pain [4]. The ECG demonstrated ST-segment elevation in leads V1-V6 consistent with acute anterior wall myocardial infarction and the patient was found to have a large tumor involving the right ventricular free wall on TTE. Another case published by Xiang et al. in February 2020 detailed a 66-year-old man who presented to his internist's office with nonproductive cough, lower extremity edema, and dyspnea at rest who was found to have elevation of the ST-segment in leads II, III, and aVF, as well as leads V3-V6 concerning for acute myocardial infarction [5]. Computed tomography of the patient's chest demonstrated anterior bulging of the left and right ventricles that was subsequently biopsied and revealed high-grade malignant spindle and epithelioid neoplasm. In contrast to these and other previously documented cases, our patient was asymptomatic from his cardiac tumor at presentation, even in the context of developing cardiac tamponade physiology on TTE. 
published by Akgun et al. in March 2020 described particular ECG features that could potentially assist in identifying the etiology and prevent inappropriate and unnecessary interventions [8]. The authors reported that of the 36 patients for whom the ECG characteristics were evaluated, 35 (97.2\%) had ST-segment elevations that corresponded to a specific coronary territory. This is consistent with the ECG findings from our patient whose pattern of injury localized to an anteroseptal distribution (i.e., left anterior descending artery), which correlated with the myocardial region where the mass was noted on echocardiogram. Interestingly, pathologic $\mathrm{Q}$ waves and/or poor $\mathrm{R}$ wave progression suggestive of infarcted myocardium were only noted in one patient in the review. The absence of these findings in our patient further supports the notion that our patient did not suffer a STEMI and, instead, the ST-segment elevation likely represented a current of injury directly related to the metastatic cardiac tumor. We were unable to further elucidate the precise pathophysiological mechanism that may have generated the current of injury as an autopsy evaluation was declined.

\section{Conclusions}

This case report describes a young, active-duty military patient with classic findings of an anteroseptal STsegment elevation myocardial infarction on ECG with elevated troponin level that ultimately appeared to be attributable to a current of injury from an interventricular septal mass. We consider this an important case to report as it reinforces the importance of maintaining a wide differential diagnosis for ST-segment elevation, particularly in the context of a patient with known metastatic malignancy and absence of typical anginal symptoms, risk factors, or signs of developing heart failure to help prevent unnecessary morbidity and mortality.

\section{Additional Information \\ Disclosures}

Human subjects: Consent was obtained or waived by all participants in this study. Conflicts of interest: In compliance with the ICMJE uniform disclosure form, all authors declare the following: Payment/services info: All authors have declared that no financial support was received from any organization for the submitted work. Financial relationships: All authors have declared that they have no financial relationships at present or within the previous three years with any organizations that might have an interest in the submitted work. Other relationships: The views expressed herein are those of the authors and do not reflect the official policy or position of Brooke Army Medical Center, the U.S. Army Medical Department, the U.S. Army Office of the Surgeon General, the Department of the Army, the Department of the Air Force, and the Department of Defense or the U.S. Government.

\section{References}

1. Hanna EB, Glancy DL: ST-segment elevation: differential diagnosis, caveats. Cleve Clin J Med. 2015, 82:373384. 10.3949/ccjm.82a.14026

2. Butany J, Leong SW, Carmichael K, Komeda M: A 30-year analysis of cardiac neoplasms at autopsy . Can J Cardiol. 2005, 21:675-680.

3. Lam KY, Dickens P, Chan AC: Tumors of the heart. A 20-year experience with a review of 12,485 consecutive autopsies. Arch Pathol Lab Med. 1993, 117:1027-1031.

4. Demir V, Turan Y, Ede H, Hidayet S, Erkoç MF: Electrocardiographic changes in right ventricular metastatic cardiac tumor mimicking acute ST elevation myocardial infarction: a case of misdiagnosis. Turk J Emerg Med. 2018, 19:33-35. 10.1016/j.tjem.2018.07.001

5. Xiang K, Bhave PD, Whalen SP, Singleton MJ: Cardiac tumor masquerading as ST elevation myocardial infarction: a case report and literature review. J Clin Cases Rep. 2020, 4:39-42.

6. Thygesen K, Alpert JS, Jaffe AS, et al.: Fourth Universal Definition of Myocardial Infarction (2018). J Am Coll Cardiol. 2018, 72:2231-2264. 10.1016/j.jacc.2018.08.1038

7. Barnes AR, Beaver DC, Snell AM: Primary sarcoma of the heart: report of a case with electrocardiographic and pathological studies. Am Heart J. 1934, 9:480-491. 10.1016/S0002-8703(34)90096-X

8. Akgun T, Gulsen K, Cinier G, et al.: Electrocardiographic characteristics of metastatic cardiac tumors presenting with ST-segment elevation. J Electrocardiol. 2020, 59:93-99. 10.1016/j.jelectrocard.2019.12.014 